\title{
Point-of-care ultrasound for the detection of hydronephrosis in emergency department patients with suspected renal colic
}

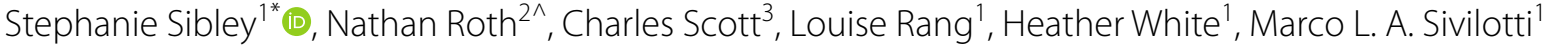

and Eric Bruder ${ }^{1}$

\begin{abstract}
Background: Point-of-care ultrasound (PoCUS) by emergency physicians for renal colic has been proposed as an alternative to computed tomography (CT) to avoid ionizing radiation exposure and shorten emergency department length of stay. Previous studies have employed experienced or credentialed ultrasonographers or required advanced ultrasound skills. We sought to measure the diagnostic accuracy of PoCUS by physicians with varied experience using a simplified binary outcome of presence or absence of hydronephrosis. Secondary outcomes include assessment as to whether the presence of hydronephrosis on PoCUS is predictive of complications, and to evaluate possible causes for the reduced diagnostic accuracy such as body mass index (BMI) and time between PoCUS and formal imaging, and scanner experience.
\end{abstract}

Results: 413 patients were enrolled in the study. PoCUS showed a specificity of $71.8 \%$ [95\% Cl 65.0, 77.9] and sensitivity of $77.1 \%$ [95\% Cl 70.9, 82.6]. Hydronephrosis on PoCUS was predictive of complications (relative risk 3.13; [95\% Cl $1.30,7.53])$. The time interval between PoCUS and formal imaging, BMI, and scanner experience did not influence the accuracy of PoCUS.

Conclusions: PoCUS for hydronephrosis in suspected renal colic has moderate accuracy when performed by providers with varied experience for the binary outcome of presence or absence of hydronephrosis. Hydronephrosis on PoCUS is associated with increased rates of complications. PoCUS for hydronephrosis is limited in its utility as a stand-alone test, however this inexpensive, readily available test may be useful in conjunction with clinical course to determine which patients would benefit from formal imaging or urologic consultation.

ClinicalTrials.gov Identifier NCT01323842

Keywords: Hydronephrosis, PoCUS, Point-of-care ultrasound, Renal colic, Emergency department, BMI

\section{Background}

Renal colic is a common presentation in the ED with over 3.6 million visits in the United States between 2006 and 2009 [1]. While most attacks are self-limited, some patients benefit from urologic intervention, and others

\footnotetext{
*Correspondence: stephanie.sibley@kingstonhsc.ca

^Nathan Roth: Deceased (19th September 2017)

${ }^{1}$ Department of Emergency Medicine, Queen's University, Kingston, ON,

Canada

Full list of author information is available at the end of the article
}

harbor a more dangerous cause for their pain. Since identifying such cases is largely based on formal imaging, especially computed tomography (CT), suspected renal colic is an important driver of advanced imaging, of prolonged or repeated emergency department utilization, and of direct and indirect costs related to patient care and lost work time [2-4].

Computed tomography remains the imaging modality of choice to confirm the diagnosis, prognosticate spontaneous stone passage, and rule out other causes of abdominal pain $[5,6]$. A study of US emergency department 
visits for suspected renal colic reported very high rates of abdominal CT $(>80 \%)$ despite low rates of admission $(<20 \%)$ and urologic intervention $(<10 \%)$ [7]. Radiology ultrasound (radUS) has been proposed as the preferred test for patients at low risk for complications of urolithiasis [6]. When using this modality, the calculus is often not visualized and the identification of hydronephrosis due to ureteric obstruction becomes the salient finding. Point-of-care ultrasound (PoCUS) by emergency physicians has been widely adopted in emergency medicine [8] and has been proposed as a cost-effective, quick and radiation-free alternative to $\mathrm{CT}$ to aid in decision-making in cases of suspected renal colic [9-17]. Several studies have reported only moderate sensitivity and specificity of PoCUS for urolithiasis. These studies have been performed by either credentialed ultrasonographers [12] or attending emergency physicians [15] or have required physicians to perform complicated scans including grading the degree of hydronephrosis and identifying ureteric jets $[10,11,14,17]$. Smith-Bindman et al. found no significant difference in high-risk diagnoses, return emergency visits, pain scores or hospitalizations when initial ultrasonography was compared to CT. However, there was no head-to-head comparison between PoCUS and $\mathrm{CT}$ in this comparative effectiveness study, the diagnosis of renal colic was dependent on passage of a stone or surgical intervention rather than formal imaging, and obese patients were excluded from this study, limiting its generalizability [18].

We performed a prospective, observational study to determine the diagnostic accuracy of PoCUS for hydronephrosis in patients with suspected renal colic. Our secondary outcome evaluated if hydronephrosis on PoCUS was predictive of patients who developed subsequent complications. This study adds to the current body of literature by addressing issues such as physician experience, generalized by having resident and attending emergency physicians with varied levels of ultrasound experience perform scans, and a simplified binary outcome of presence or absence of hydronephrosis as opposed to grading hydronephrosis or detecting ureteric jets. Additionally, the effect of body mass index (BMI), scanner experience and the time interval from formal imaging to PoCUS on the accuracy of this test was assessed.

\section{Methods}

This was a prospective observational study conducted in two academic emergency departments in Ontario (combined visits of $\sim 110,000 /$ year). The Queen's University Health Sciences Research Ethics Board approved this study, which accrued subjects over 30 months (April 2011-July 2013). All subjects provided written consent prior to enrollment.
Patients aged $16-65$ years who had either CT or radUS ordered for suspected renal colic were screened for eligibility. The treating emergency physicians enrolled eligible patients $24 \mathrm{~h}$ a day, 7 days a week, assisted by dedicated research personnel during daytime to evening hours up to 7 days a week, which matched the availability of advanced imaging for this indication.

Patients were excluded for hemodynamic instability, fever, suspected urinary tract infection based on symptoms or urinalysis positive for leukocytes and nitrites, pregnancy, renal transplant, single functioning kidney, known abdominal aortic aneurysm or incarceration. Patients were ineligible if no formal imaging was ordered at the time of their emergency visit.

Attending or resident emergency physicians performed the PoCUS scans. To be eligible to perform study scans, physicians had to complete an accredited emergency department ultrasound course (such as those endorsed by the Canadian Point of Care Ultrasound Society) or the local, required, introductory point-of-care ultrasound course for emergency medicine residents focusing on focused assessment with sonography in trauma (FAST), aortic and obstetrical ultrasound skills. Scanning physicians had to attend a didactic lecture where the study was described and renal anatomy, ultrasound technique and several examples were reviewed. They attended a training session where physicians scanned live models with hydronephrosis. The newly trained physicians had to complete 25 observed renal ultrasounds with an expert physician (fellowship trained or additional training in renal ultrasound) prior to enrolling patients.

Other than undergoing PoCUS, subjects were treated according to usual practice. The decision and type of formal imaging was left entirely to the discretion of the treating physician, and potential subjects were approached by research staff only after formal imaging had been ordered. Scans were performed using a My Lab 5 (Esaote, Genoa Italy) ultrasound machine with a curvilinear 3.5-MHz probe. Scanning physicians performed a B-mode scan of the abdominal aorta and bilateral kidneys (short and long axis). The scanning physician recorded subject weight, height, if hydronephrosis was present/absent/indeterminate for each kidney, as well as the diameter of the abdominal aorta on a standardized form while blinded to any formal imaging. In cases where the formal imaging was performed prior to PoCUS, the scanning physician was directed not to look at images or the formal radiology report.

A radiologist (CS) blinded to the PoCUS reviewed all formal CT or radUS images to establish the degree of hydronephrosis, graded as absent, mild, moderate or severe [19]. The radiologist also documented the presence, position and size of any urinary calculus, signs 
suggestive of recent stone passage, as well as any alternative diagnosis that could account for the patient's symptoms and the presence of abdominal aortic aneurysm (AAA) on a standardized form.

Research assistants performed 30-day telephone follow-up and asked scripted standardized questions regarding urgent consultations with urologists, any interventions or hospitalizations. A chart review was performed at 30 days (NR and SS). Details of urologic consultations, interventions, hospitalizations, sepsis and death within the regional healthcare system were extracted from the record, recorded on standardized forms, and entered into a RedCap database. Patients were considered lost to follow-up if they could not be contacted by telephone and had no record of emergency room visit, urology intervention or hospital admission in the medical record.

The primary outcome measure was the sensitivity and specificity of PoCUS for the presence or absence of hydronephrosis compared to formal imaging in patients with suspected renal colic.

The secondary outcome was the association between PoCUS findings and complications within 30 days, defined as the composite of any urologic intervention (e.g., lithotripsy, stent, or percutaneous nephrostomy), sepsis, hospital admission or death. Urgent complications were defined as any of the composite outcomes that occurred within 7 days of initial emergency department visit. Urgent complications were assessed separately to differentiate patients who presented with an acute worsening of their symptoms versus patients who had urology follow-up and were scheduled for a surgical procedure within 30 days. The effects of BMI, time interval to formal imaging and physician scanning experience on the accuracy of PoCUS were analyzed.

For the primary analysis, indeterminate PoCUS scans were deemed negative for hydronephrosis, but reclassified as positive in a separate sensitivity analysis. The degree of severity of hydronephrosis on formal imaging was dichotomized at different cut points to determine the diagnostic accuracy of PoCUS when hydronephrosis was considered positive only when graded "mild, moderate and severe", "moderate and severe", or "severe". Patients who were lost to 30-day follow-up were excluded from the analysis for the secondary outcome of complications and no imputation was performed.

Statistical analysis was performed SAS software, version 9.4 (Cary, North Carolina). Baseline characteristics were summarized as means and standard deviations and medians and quartiles for continuous variables and proportions for binary and categorical variables. Sensitivity, specificity and likelihood ratios were calculated for detection of hydronephrosis with PoCUS using the formal radiology result as the reference standard. A Chi square test was used to compare risk of complications, and to assess for changes in accuracy for detection of hydronephrosis compared to formal imaging time between PoCUS and formal imaging, and BMI.

Results are reported in accordance with the STARD 2015 guidelines for studies of diagnostic accuracy [20].

We performed an a priori sample size calculation using estimates taken from Edmonds et al. [21], a retrospective chart review of patients undergoing formal ultrasound for the diagnosis of renal colic in emergency patients. These investigators reported that $0.6 \%$ of subjects with no abnormality on ultrasound received urologic intervention versus $6.2 \%$ of patients with a visualized stone and $6.8 \%$ of patients with ultrasounds suggestive of ureterolithiasis. Assuming a 7\% versus 1\% in complications/interventions between patients with and without hydronephrosis on PoCUS, two-tailed $\alpha$ of $5 \%$, and a power of $80 \%$, it was determined that 167 subjects would be required in each group. We estimated that this would provide $95 \%$ confidence bands of $\pm 10 \%$ around the point estimate of sensitivity and specificity for the primary outcome.

\section{Results}

A total of 955 unique patient encounters were screened for potential enrollment in the study. The total enrollment was 415 patients, and 413 patients completed formal imaging and represent the study cohort for the primary analysis of hydronephrosis. Total patients lost at 1-month follow-up totaled 69, leaving 344 patients in the analysis for the secondary outcome of urologic complications (Fig. 1).

Seventeen emergency physicians and residents were eligible to perform renal PoCUS for the study, including five physicians considered to be experts.

Baseline characteristics of the cohort are summarized in Table 1 . Half $(50.4 \%)$ were male, with mean age was 42.2 years and mean BMI was $29 \mathrm{~kg} / \mathrm{m}^{2}$. Most patients (85.0\%) underwent formal imaging with a CT scan. Hydronephrosis on formal imaging was found in $51.0 \%$ of patients. Hydronephrosis on PoCUS was seen in 53.0\% of patients, and $4.6 \%$ had indeterminate PoCUS scans. A small number of subjects had alternate diagnoses established on formal imaging, including ovarian cysts, diverticulitis and appendicitis (Table 2). Of those with an alternate diagnosis the detection of hydronephrosis with POCUS was rare and seen only in one case of pyelonephritis, one case of perforated diverticulitis, one ureteric stricture and one gynecological cancer. There were no cases of ectopic pregnancy or abdominal aortic aneurysm in the study cohort. 


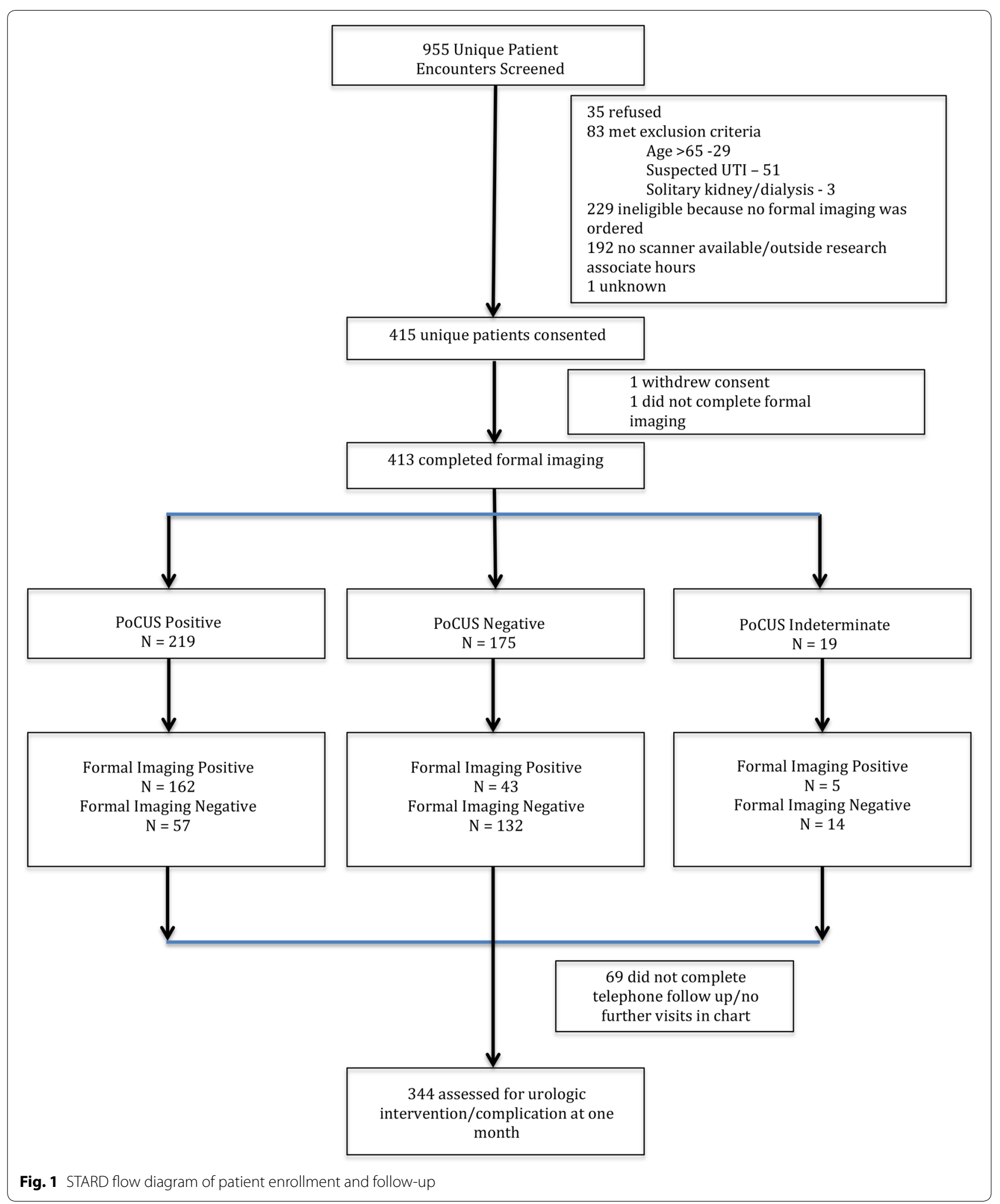


Table 1 Cohort characteristics

\begin{tabular}{|c|c|}
\hline $\mathbf{N}$ & 413 \\
\hline Male (\%) & $208(50.4)$ \\
\hline Age, years mean (SD) & $42.2(13.5)$ \\
\hline BMI, mean (SD) & $28.96(11.2)$ \\
\hline \multicolumn{2}{|l|}{ BMI category (\%) } \\
\hline$<20$ & $27(6.7)$ \\
\hline $20-24.9$ & $111(27.6)$ \\
\hline $25-29.9$ & $126(31.3)$ \\
\hline $30-34.9$ & $76(18.9)$ \\
\hline$>35$ & $62(15.4)$ \\
\hline Missing & 10 \\
\hline \multicolumn{2}{|l|}{ Time from POCUS to formal imaging, min } \\
\hline Mean (SD) & $-31.81(63.1)$ \\
\hline Median & -40.50 \\
\hline 25th percentile & -58.0 \\
\hline 75th percentile & -24.0 \\
\hline \multicolumn{2}{|l|}{ Hydronephrosis present on POCUS } \\
\hline No & $175(42.4)$ \\
\hline Yes & $219(53.0)$ \\
\hline Indeterminate & $19(4.6)$ \\
\hline \multicolumn{2}{|l|}{ Formal imaging modality } \\
\hline $\mathrm{CT}$ & $350(85.0)$ \\
\hline US & $63(15.0)$ \\
\hline \multicolumn{2}{|l|}{ Hydronephrosis on formal imaging } \\
\hline Severe & $19(4.6)$ \\
\hline Moderate & $78(19.0)$ \\
\hline Mild & $113(27.4)$ \\
\hline Absent & $202(49.0)$ \\
\hline 2nd ER visit for Imaging results (\%) & $49(12)$ \\
\hline Urologic Consult & $60(17.4)$ \\
\hline \multicolumn{2}{|l|}{ Urologic intervention/complication } \\
\hline Lithotripsy & $15(4.4)$ \\
\hline Stent & $20(5.8)$ \\
\hline Percutaneous nephrostomy & 0 \\
\hline Sepsis & $8(2.3)$ \\
\hline Hospital admission & $17(5.2)$ \\
\hline Death & 0 \\
\hline $\begin{array}{l}\text { Stone or evidence of recent passage seen on formal } \\
\text { imaging (\%) }\end{array}$ & $205(50)$ \\
\hline
\end{tabular}

SD standard deviation, BMI body mass index, CT computed tomography, US ultrasound)

Twenty-eight (6.8\%) subjects developed the composite outcome at 30 days, with 19 (4.6\%) deemed to be urgent within 7 days. There were no deaths.

The sensitivity of POCUS for the detection of hydronephrosis was $77.1 \%$ [95\% CI 70.9, 82.6] and the specificity was $71.8 \%$ [95\% CI 65.0, 77.9]. The sensitivity of PoCUS improved with worsening degrees of hydronephrosis. Two unplanned sensitivity analyses were performed.
Table 2 Alternative diagnoses found on formal imaging

\begin{tabular}{ll}
\hline Ovarian cyst & 6 \\
Diverticulitis & 4 \\
Appendicitis & 3 \\
Pyelonephritis & 3 \\
Biliary colic/cholecystitis & 3 \\
Ovarian torsion/mass & 2 \\
Bony lesions/compression fracture & 2 \\
Epiploic appendagitis & 2 \\
Shingles & 2 \\
Uterine mass/cancer & 2 \\
Testicular torsion & 1 \\
Interabdominal carcinomatosis with metastatic disease to the liver & 1 \\
Pneumonia & 1 \\
Bowel obstruction & 1 \\
lleitis & 1 \\
AAA & 0 \\
Ectopic pregnancy & 0
\end{tabular}

$A A A$ abdominal aortic aneurysm

These measures did not improve appreciably for patients with confirmed stone or signs of recent stone passage on formal imaging (sensitivity 78.2 [95\% CI 71.3, 84.1], specificity 74.2 [95\% CI 55.4, 88.1]), or when considering only patients who had a CT scan (sensitivity 75.9 [95\% CI 69.2, 81.8], specificity 72.3 [95\% CI 64.7, 79.1]) (Table 3).

The presence of hydronephrosis on PoCUS was associated with increased complications (11.8\% [95\% CI 7.6, 17.4] versus $3.8 \%$ [95\% CI 1.4, 8.0], relative risk of 3.1 [95\% CI 1.3, 7.5]) (Table 4). The association between hydronephrosis on formal imaging and 30-day complications was somewhat stronger $(14.5 \%$ [95\% CI 9.7, 20.6] versus $1.21 \%$ [ $95 \% \mathrm{CI} 0.2,4.3$ ], relative risk 11.98 [95\% CI $2.9,49.7])$.

In a post hoc analysis, we considered only the subgroup of patients with urgent interventions. The presence of hydronephrosis on PoCUS was also associated with urgent interventions within 7 days $(8.1 \%$ [95\% CI $4.6,13.0]$ versus $2.5 \%$ [ $95 \%$ CI $0.7,6.3]$, relative risk of 3.2 [95\% CI 1.1, 9.5]).

A sensitivity analysis was performed where indeterminate scans were considered positive, with little difference in complications $(11.4 \%$ [95\% CI 7.0, 15.7] vs 3.5\% [95\% CI 0.4, 6.6], relative risk 3.23\% [95\% CI 1.3, 8.3]).

The diagnostic accuracy of PoCUS for hydronephrosis did not appear to be strongly influenced by any of the pre-specified factors we examined. Almost all PoCUS scans were obtained within $1 \mathrm{~h}$ of formal imaging (Fig. 2). Most (86.2\%) patients had PoCUS performed after being transferred back to the emergency department from formal imaging. The agreement did 
Table 3 Sensitivity, specificity, positive-likelihood ratio and negative-likelihood ratio for detection of hydronephrosis with POCUS

\begin{tabular}{|c|c|c|c|c|c|c|c|c|}
\hline & TP & TN & FP & FN & Sensitivity $(95 \% \mathrm{Cl})$ & Specificity $(95 \% \mathrm{Cl})$ & $+\mathrm{LR}(95 \% \mathrm{Cl})$ & $-\mathrm{LR}(95 \% \mathrm{Cl})$ \\
\hline $\begin{array}{l}\text { Hydronephrosis (mild/moderate/severe } \\
\text { positive) }^{\mathrm{a}}\end{array}$ & 162 & 146 & 57 & 48 & $77.1 \%(70.9,82.6)$ & $71.8 \%(65.0,77.9)$ & $2.73(2.17,3.48)$ & $0.31(0.24,0.41)$ \\
\hline Hydronephrosis (moderate/severe positive) ${ }^{\mathrm{a}}$ & 85 & 182 & 134 & 12 & $87.6(79.4,93.4)$ & $57.5(51.8,63.0)$ & $2.05(1.78,2.39)$ & $0.22(0.13,0.37)$ \\
\hline Hydronephrosis (severe positive) $^{\mathrm{a}}$ & 18 & 193 & 201 & 1 & $94.7(74.0,99.9)$ & $48.9(43.8,53.9)$ & $1.85(1.60,2.14)$ & $0.11(0.02,0.73)$ \\
\hline $\begin{array}{l}\text { Hydronephrosis (with confirmed stone on } \\
\text { formal imaging) }^{\mathrm{b}}\end{array}$ & 136 & 23 & 8 & 38 & $78.2(71.3,84.1)$ & $74.2(55.4,88.1)$ & $3.03(1.66,5.53)$ & $0.29(0.21,0.42)$ \\
\hline Hydronephrosis (patients undergoing CT scan) ${ }^{c}$ & 145 & 115 & 44 & 46 & $75.9(69.2,81.8)$ & $72.3(64.7,79.1)$ & $2.74(2.11,3.57)$ & $0.33(0.25,0.44)$ \\
\hline
\end{tabular}

$L R$ likelihood ratio, $T P$ true positive, $T N$ true negative, $F P$ false positive, $F N$ false negative)

${ }^{a}$ A positive test on formal imaging was dichotomized at various cut points (mild/moderate/severe, moderate/severe, severe)

b Sensitivity analysis with confirmed stone on formal imaging

c Senstivity analysis with the gold standard of CT scan

Table 4 Intervention/complication for POCUS and formal imaging

\begin{tabular}{|c|c|c|c|c|}
\hline & $\begin{array}{l}\text { Intervention/ } \\
\text { complication }\end{array}$ & $\begin{array}{l}\text { No intervention/ } \\
\text { complication }\end{array}$ & Risk (95\% Cl) & Relative risk $(95 \% \mathrm{Cl})$ \\
\hline \multicolumn{5}{|l|}{ POCUS } \\
\hline Positive & 22 & 163 & $11.83 \%(7.56,17.36)$ & $3.13(1.30,7.53)$ \\
\hline Negative/indeterminate & 6 & 153 & $3.77 \%(1.40,8.03)$ & \\
\hline \multicolumn{5}{|l|}{ POCUS } \\
\hline Positive/indeterminate & 23 & 179 & $11.39 \%(7.36,16.59)$ & $3.23(1.26,8.30)$ \\
\hline Negative & 5 & 137 & $3.52 \%(1.15,8.03)$ & \\
\hline \multicolumn{5}{|l|}{ Formal imaging } \\
\hline Positive & 26 & 153 & $14.53 \%(9.71,20.55)$ & $11.98(2.89,49.70)$ \\
\hline Negative & 2 & 163 & $1.21 \%(0.15,4.31)$ & \\
\hline
\end{tabular}

Patients were positive for intervention/complication if they had any one of the following at 30 days follow-up-lithotripsy, stent, percutaneous nephrostomy, sepsis, hospital admission or death

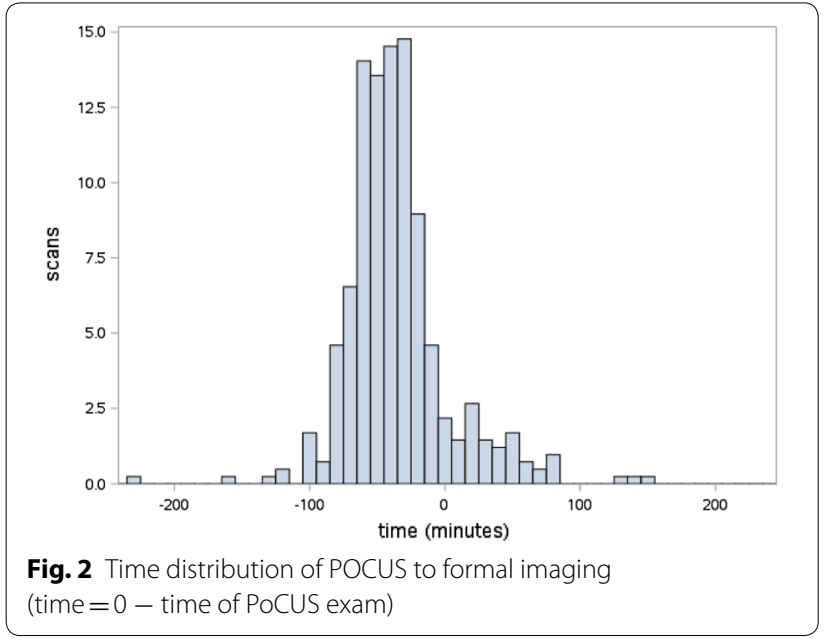

not improve when the absolute value of the time interval between formal imaging and PoCUS was shorter. Agreement with formal imaging for each quartile was $76.2 \%, 78.1 \%, 76.5 \%$ and $67.3 \%(p=0.27)$.

When performing PoCUS for the detection of hydronephrosis, expert scanners were comparable to nonexperts (sensitivity $81.5 \%$ [95\% CI 70.0, 90.1] vs $75.2 \%$ [95\% CI 67.3, 82.0]), (specificity 65.4 [95\% CI 50.9, 78.0] vs 74.2 [95\% CI 66.4, 80.9]). There was no difference in agreement with formal imaging, with experts in agreement $66.7 \%$ [95\% CI 57.4, 75.1] and non-experts in agreement 63.9\% [95\% CI 58.1, 69.3] $(p=0.65)$. Agreement between PoCUS and formal imaging did not improve with a physician's increased scanning experience within the study Agreement was $70.1 \%$ for $1-3$ 


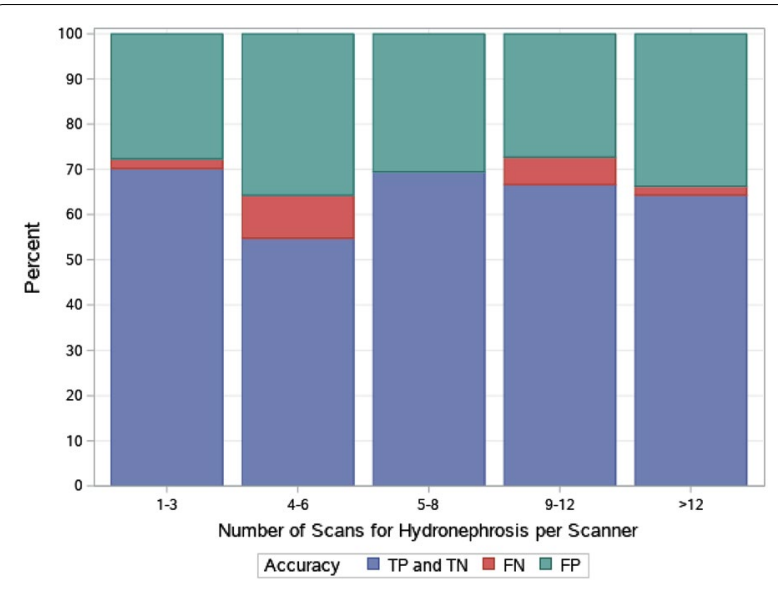

Fig. 3 Accuracy of POCUS with formal imaging with increasing scanning experience (TP true positive, $T N$ true negative, FP false positive, FN false negative)

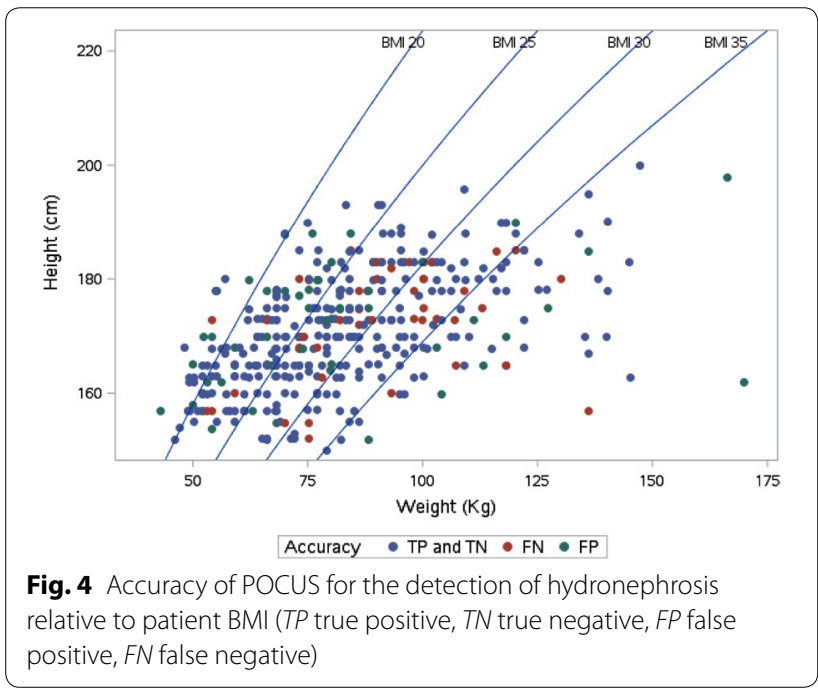

scans, $54.8 \%$ for $4-6$ scans, $69.4 \%$ for $10-12$ scans, and $64.3 \%$ for $>12$ scans (Fig. 3 ).

BMI did not influence the accuracy with agreement between PoCUS and formal imaging of $70.4 \%, 73.9 \%$, $72.2 \%$ and $81.6 \%$ and $71.0 \%$ for BMI of $<20,20-24.9$, $25-29.9,30-34.9$ and $>30$, respectively $(p=0.56)$ (Fig. 4).

\section{Discussion}

Our study reaffirms the modest sensitivity and specificity reported by previous studies which limits the utility of PoCUS as a stand-alone diagnostic tool for renal colic. PoCUS is predictive of complications and may help identify patients who require further imaging and urologic consultation.
Our study has similar results to a meta-analysis [9] by Wong et al. that demonstrated modest diagnostic accuracy for nephrolithiasis with moderate sensitivity (77.1\%) and specificity (71.8\%). They included highquality studies $(N=1773)$ including two studies with scans performed by credentialed ultrasonographers [12] or attending emergency physicians [15] and four studies requiring physicians to perform more complicated scans $[10,11,14,17]$. While these studies showed improved specificity, physician ability to grade hydronephrosis is moderate and limits sensitivity [22]. Our study demonstrated that physicians at all levels of training and experience can obtain similar sensitivity and specificity while performing a simplified scan for the presence or absence of hydronephrosis.

The presence of hydronephrosis on PoCUS was associated with an increased risk of subsequent urologic intervention or complication in our study. Fields et al. [11] performed a study of 77 emergency department patients to predict 30-day outcomes. Thirteen patients required admission to hospital, and all admitted patients had hydronephrosis on their PoCUS exam. Daniels et al. [10] performed a study to determine the ability of PoCUS to predict need for urologic intervention at 90 days and demonstrated that $22.8 \%$ of patients with hydronephrosis as opposed to $10 \%$ of those without required urologic intervention.

While PoCUS was associated with need for urologic intervention, it identified fewer patients than $\mathrm{CT}$ or radUS. This is in keeping with a study by Ganesan et al. suggesting $22 \%$ of patients would be inappropriately counseled for either observation or intervention if ultrasound was used alone to guide clinical decision-making [23].

Smith-Bindman et al. showed PoCUS has the advantage of being a readily available modality that has the potential to reduce the time from presentation to imaging in the emergency department and expedite clinical decision-making [18]. In this study, we hypothesized the modest sensitivity and specificity of PoCUS in previous studies may have been due to the length of time between PoCUS and formal imaging due to factors such as dehydration and fluid administration [24], and spontaneous stone passage [25]. PoCUS was performed as close to the formal imaging as possible to reduce the effect of these factors. Most of the PoCUS scans were performed within $1 \mathrm{~h}$ of formal imaging and there was no difference in agreement with shorter time intervals, indicating these factors had little influence on the sensitivity and specificity in this study.

There was no difference in accuracy between expert and non-expert scanners and no improvement with increased scanning experience which suggests the training session 
and 25 practice scans prior to enrolling patients in the study may be adequate to develop and retain the skill for detection of hydronephrosis. This is in contrast to Herbst et al. who found fellowship trained emergency physicians had greater accuracy than those without fellowship training [14]. This difference is likely a result of the differing definition of expert scanners and the outcome of graded hydronephrosis versus a simplified binary outcome. It is important to note the overall sensitivity (72.6\%), specificity $(73.3 \%)$ were similar.

Few PoCUS studies have assessed the relationship between diagnostic accuracy and BMI for detection of renal colic. BMI was not a predictor of accuracy of PoCUS for hydronephrosis in this study, and goodquality images were obtained in patients with BMI up to $35 \mathrm{~kg} / \mathrm{m}^{2}$. This is in keeping with findings using ultrasound by diagnostic imaging sonographers $[23,26]$, who found that detection of a renal stone was independent of BMI.

While PoCUS for hydronephrosis lacks the sensitivity and specificity for a stand-alone diagnostic test, it may be useful when combined with a clinical prediction tool to improve accuracy in diagnosis [10] or clinical score based on history and physical exam [27]. The ability to use this test in patients with increased risk factors such as older age, fever, leukocytosis, history of kidney disease or solitary kidney remains unclear.

\section{Strengths and limitations}

The strengths of this study are the inclusion of both resident and attending physicians with varied PoCUS experience and our comprehensive renal PoCUS training program which included practice scanning on patients with known hydronephrosis and observed scans by PoCUS experts. This study also included obese patients who were excluded from other large studies.

Limitations to our study include missed, potentially eligible patients due to lack of an available scanning physician which may have introduced a selection bias. As well, many patients had their PoCUS exam performed after their formal imaging was completed, however scanners were instructed not to look at any imaging prior to completing the PoCUS exam. Most of the PoCUS exams were completed within $1 \mathrm{~h}$ of the formal imaging, and it is unlikely the formal reports would be available prior to the completion of the PoCUS exam, mitigating this risk of unblinding. Finally, radUS is not considered a diagnostic gold standard for detection of renal stones or recent passage. $15 \%$ of patients had formal ultrasound as opposed to CT scanning, mainly young women. A sensitivity analysis was performed for patients who received a CT scan and little difference in sensitivity and specificity was noted.

\section{Conclusion}

PoCUS for hydronephrosis has moderate sensitivity and specificity for renal colic limiting its utility as a diagnostic test. However, patients with hydronephrosis are more likely to develop complications, and PoCUS may be useful to help guide further imaging and consultation in conjunction with clinical course. BMI, provider experience and time between PoCUS and formal imaging do not influence the accuracy of this test.

\section{Acknowledgements}

Special thanks to Dr. Andrew Day and Dr. Xiaoqun Sun for their assistance with statistical analysis, to Jane Reid and the research coordinators for screening for patients and to Dr. Heather Mackenzie and Dr. Arianne Buchan for support.

\section{Authors' contributions}

$S S, L R$, EB and MS conceived and designed the study, obtained funding and supervised the conduct of the study. SS, NR and HW performed chart reviews and data entry. CS performed the formal radiology assessments. SS performed the statistical analysis and drafted the manuscript with substantial input from MS. SS takes responsibility for the paper as a whole. All authors read and approved the final manuscript.

\section{Funding}

This study was funded through a SEAMO Innovation Grant. SEAMO had no involvement in the design of the study or collection, analysis, interpretation of data or writing the manuscript.

\section{Availability of data and materials}

All data collection forms are stored in the Department of Emergency Medicine Research Office. The RedCap database used for electronic entry is housed at Queen's University Clinical Evaluation Research Unit. This data is not publicly available due to confidentially issues, however, de-identified data are available from the corresponding author on reasonable request.

\section{Ethics approval and consent to participate}

The Queen's University Health Sciences Research Ethics Board approved this study. Written consent was obtained from all patients enrolled in the study.

Consent for publication

Written consent was obtained from all patients enrolled in the study.

\section{Competing interests}

There are no conflicts of interest to declare.

\section{Author details}

1 Department of Emergency Medicine, Queen's University, Kingston, ON, Canada. ${ }^{2}$ School of Medicine, Faculty of Health Sciences, Queen's University, Kingston, ON, Canada. ${ }^{3}$ Department of Radiology, Queen's University, Kingston, ON, Canada.

Received: 31 January 2020 Accepted: 26 May 2020

Published online: 08 June 2020

\section{References}

1. Ghani KR, Roghmann F, Sammon JD et al (2014) Emergency department visits in the United States for upper urinary tract stones: trends in hospitalization and charges. J Urol 191:90-96

2. Saigal CS, Joyce G, Timilsina AR, Urologic Diseases in America P (2005) Direct and indirect costs of nephrolithiasis in an employed population: opportunity for disease management? Kidney Int 68:1808-1814

3. Eaton SH, Cashy J, Pearl JA, Stein DM, Perry K, Nadler RB (2013) Admission rates and costs associated with emergency presentation of urolithiasis: analysis of the Nationwide Emergency Department Sample 2006-2009. J Endourol 27:1535-1538 
4. Pearle MS, Calhoun EA, Curhan GC, Urologic Diseases of America P (2005) Urologic diseases in America project: urolithiasis. J Urol 173:848-857

5. Brisbane W, Bailey MR, Sorensen MD (2016) An overview of kidney stone imaging techniques. Nat Rev Urol 13:654-662

6. Wang RC (2016) Managing urolithiasis. Ann Emerg Med 67:449-454

7. Schoenfeld EM, Pekow PS, Shieh MS, Scales CD Jr, Lagu T, Lindenauer PK (2017) The diagnosis and management of patients with renal colic across a sample of US Hospitals: high CT utilization despite low rates of admission and inpatient urologic intervention. PLoS ONE 12:e0169160

8. American College of Emergency P (2009) Emergency ultrasound guidelines. Ann Emerg Med 53:550-570

9. Wong C, Teitge B, Ross M, Young P, Robertson HL, Lang E (2018) The accuracy and prognostic value of point-of-care ultrasound for nephrolithiasis in the emergency department: a systematic review and meta-analysis. Acad Emerg Med 25(6):684-698

10. Daniels B, Gross CP, Molinaro A et al (2016) STONE PLUS: evaluation of emergency department patients with suspected renal colic, using a clinical prediction tool combined with point-of-care limited ultrasonography. Ann Emerg Med 67:439-448

11. Fields JM, Fischer Jl, Anderson KL, Mangili A, Panebianco NL, Dean AJ (2015) The ability of renal ultrasound and ureteral jet evaluation to predict 30-day outcomes in patients with suspected nephrolithiasis. Am J Emerg Med 33:1402-1406

12. Gaspari RJ, Horst K (2005) Emergency ultrasound and urinalysis in the evaluation of flank pain. Acad Emerg Med 12:1180-1184

13. Goertz JK, Lotterman S (2010) Can the degree of hydronephrosis on ultrasound predict kidney stone size? Am J Emerg Med 28:813-816

14. Herbst MK, Rosenberg G, Daniels B et al (2014) Effect of provider experience on clinician-performed ultrasonography for hydronephrosis in patients with suspected renal colic. Ann Emerg Med 64:269-276

15. Moak JH, Lyons MS, Lindsell CJ (2012) Bedside renal ultrasound in the evaluation of suspected ureterolithiasis. Am J Emerg Med 30:218-221

16. Riddell J, Case A, Wopat R et al (2014) Sensitivity of emergency bedside ultrasound to detect hydronephrosis in patients with computed tomography-proven stones. West J Emerg Med 15:96-100

17. Watkins S, Bowra J, Sharma P, Holdgate A, Giles A, Campbell L (2007) Validation of emergency physician ultrasound in diagnosing hydronephrosis in ureteric colic. Emerg Med Australas 19:188-195
18. Smith-Bindman R, Aubin C, Bailitz J et al (2014) Ultrasonography versus computed tomography for suspected nephrolithiasis. N Engl J Med 371:1100-1110

19. Narita T, Hatakeyama S, Koie T et al (2017) Presence of transient hydronephrosis immediately after surgery has a limited influence on renal function 1 year after ileal neobladder construction. BMC Urol 17:72

20. Bossuyt PM, Reitsma JB, Bruns DE et al (2015) STARD 2015: an updated list of essential items for reporting diagnostic accuracy studies. BMJ 351:h5527

21. Edmonds ML, Yan JW, Sedran RJ, McLeod SL, Theakston KD (2010) The utility of renal ultrasonography in the diagnosis of renal colic in emergency department patients. Cjem 12:201-206

22. Pathan SA, Mitra B, Mirza S et al (2018) Emergency physician interpretation of point-of-care ultrasound for identifying and grading of hydronephrosis in renal colic compared with consensus interpretation by emergency radiologists. Acad Emerg Med 25:1129-1137

23. Ganesan V, De S, Greene D, Torricelli FC, Monga M (2017) Accuracy of ultrasonography for renal stone detection and size determination: is it good enough for management decisions? BJU Int 119:464-469

24. Morse JW, Hill R, Greissinger WP, Patterson JW, Melanson SW, Heller MB (1999) Rapid oral hydration results in hydronephrosis as demonstrated by bedside ultrasound. Ann Emerg Med 34:134-140

25. Perry KJ, Ko P, Mariani P, Ciaccio AJ (2010) Time course resolution of hydronephrosis with spontaneous ureteral stone passage. West J Emerg Med 11:105

26. Ulusan S, Koc Z, Tokmak N (2007) Accuracy of sonography for detecting renal stone: comparison with CT. J Clin Ultrasound 35:256-261

27. Aubrey-Bassler FK, Lee SD, Barter RB, Asghari S, Cullen R, Godwin M (2013) Utility of computed tomography and derivation and validation of a score to identify an emergent outcome in 2,315 patients with suspected urinary tract stone. CJEM 15:261-269

\section{Publisher's Note}

Springer Nature remains neutral with regard to jurisdictional claims in published maps and institutional affiliations.

\section{Submit your manuscript to a SpringerOpen ${ }^{\circ}$ journal and benefit from:}

- Convenient online submission

- Rigorous peer review

- Open access: articles freely available online

- High visibility within the field

- Retaining the copyright to your article

Submit your next manuscript at springeropen.com 